\title{
Low Test Results
}

National Cancer Institute

\section{Source}

National Cancer Institute. Low Test Results. NCI Thesaurus. Code C63045.

Test results provided by the device are too low or lower than expected. 